\title{
Thermodynamics Study of Solvent Adsorption on Octadecyl-Modified Silica
}

\author{
Szymon Bocian · Jan Soukup · Pavel Jandera • \\ Bogusław Buszewski
}

Received: 23 June 2014 / Revised: 30 September 2014 / Accepted: 7 October 2014 / Published online: 30 October 2014

(C) The Author(s) 2014. This article is published with open access at Springerlink.com

\begin{abstract}
Elution and solvation processes in liquid chromatography may be controlled by temperature changes. In the case of solvent adsorption, the temperature influences the amount of adsorbed solvent as well as the enthalpy and entropy of the solvation process. In this work, the thermodynamic parameters of organic solvents used as organic modifiers in the reversed-phase high-performance liquid chromatography elution process were determined. The changes of enthalpy and entropy in a series of chemically bonded stationary phases were measured to determine the effects of the temperature and surface coverage density of octadecyl ligands on the thermodynamic parameters of the solvation. For both the enthalpy and entropy a parabolic trend was observed with the minimum for medium surface coverage. The correlation of solvent adsorption values with the enthalpy of solvation was also investigated. The highest influence of the temperature on solvation process was observed for stationary phases with high surface coverage.
\end{abstract}

Keywords Column liquid chromatography · Solvent adsorption $\cdot$ Temperature influence $\cdot$ Enthalpy Entropy

S. Bocian $(\varangle) \cdot$ J. Soukup $\cdot$ B. Buszewski

Department of Environmental Chemistry and Bioanalytics, Faculty of Chemistry, Nicolaus Copernicus University, Gagarin 7 St, 87-100 Torun, Poland

e-mail: bocian@chem.umk.pl

B. Buszewski

e-mail: bbusz@chem.umk.pl

J. Soukup · P. Jandera

Department of Analytical Chemistry, Faculty of Chemical

Technology, University of Pardubice, Pardubice, Czech Republic

\section{Introduction}

When a mixed mobile phase is in contact with the hydrophobic surface of the stationary phase, less-polar solvent molecules can preferentially adsorb on the surface. The composition of the mobile phase at the surface is different from its bulk composition [1-3]. In reversed-phase liquid chromatography system, the acting stationary phase is a combination of three components: bonded ligands, residual silanols and adsorbed molecules of both organic solvent and water [4-6].

Many studies were conducted to explore the retention mechanism in reversed-phase liquid chromatography (RPLC) and two main theories were taken into account [7]. Retention mechanism in RPLC has been described by solvophobic theory introduced by Horvath and co-workers $[8,9]$ suggesting that the retention is especially influenced by hydrophobic interactions between the mobile phase and solutes and is considered to occur through non-localized adsorption rather than partitioning. The driving force for retention as described by solvophobic theory is the free energy change associated with the two-step solute-transfer mechanism including creation of a solute-sized cavity in the mobile phase and transfer of the solute to or from this cavity. The free energy change can be quantified by measuring the partition coefficients of non-polar molecules between water and non-polar solvents.

In a more recent partitioning model, Dill [10] has proposed a three-step process involving creation of a solutesized cavity in the stationary phase, transfer of the solute from the mobile phase to the stationary phase, and closing of the solute-sized cavity in the mobile phase. The solute is approximated to be fully embedded in the stationary-phase chains rather than adsorbed on the surface and this model also supposes that the retention process is especially driven 
by two forces. One is the difference in the contact-free energy of the solute in the mobile phase and the stationary phase. The second driving force for retention is related to the partial ordering of the non-polar groups bonded onto the surface of stationary phases, leading to entropic ejection of the solute from the stationary phase at sufficiently high bonding density. At low bonding densities, the partitioning of solutes should increase linearly up to a critical bonding density of about $2.7 \mu \mathrm{mol} \mathrm{m}{ }^{-2}$ suggested by Cole and Dorsey [11].

It is commonly believed that retention in the RPLC is controlled by the distribution of solute between the bulk mobile phase and the stationary phase and interactions of ligands bonded on stationary-phase surface with solute are primarily influenced by hydrophobic effect proposed. The concentration of mobile phase at the surface of stationary phase varies from its bulk concentration [3]. The residual silanols may also play very important role in adsorption of solute [6]. Indeed the retention of solute is governed by partitioning between the layer of bonded non-polar groups onto the stationary phase or by adsorption or their combination [12].

The partition coefficients can be transformed to free energy $(\Delta G)$ of transfer involving enthalpic $(\Delta H)$ and entropic $(\Delta S)$ components.

$\Delta G=\Delta H-T \Delta S$

These components may be experimentally determined by calorimetry which has been reported frequently in the literature [13]. The relationship between the free energy, retention factor, $k$, and thermodynamic temperature, $T$ (in Kelvin) [14], is described according to the following expression (van't Hoff equation) [9]:

$$
\begin{aligned}
\ln k_{i}= & \ln K+\ln \frac{V_{\mathrm{S}}}{V_{\mathrm{M}}}=\frac{-\Delta G^{0}}{\mathrm{RT}}+\ln \frac{V_{\mathrm{S}}}{V_{\mathrm{M}}}=\frac{\Delta S^{0}}{R} \\
& +\ln \frac{V_{\mathrm{S}}}{V_{\mathrm{M}}}-\frac{\Delta H^{0}}{\mathrm{RT}}=A_{\mathrm{i}}+\left(\frac{B_{\mathrm{i}}}{T}\right)
\end{aligned}
$$

In this case, the $\ln k$ versus $T^{-1}$ plots should embody linearity [15-17]. The parameter $B_{i}$ involves the standard partial molar enthalpy of transfer of the solute $i$ from the mobile phase to the stationary phase, $-\Delta H^{0}$. The parameter $A_{i}$ is proportional to the standard partial molar entropy of the transfer of the solute from the mobile phase to the stationary phase, $\Delta S^{0}$, and includes the phase ratio (the ratio of the volumes of the stationary, $V_{\mathrm{S}}$, and of the mobile, $V_{\mathrm{M}}$ ) in the chromatographic system as well [18]. The $V_{\mathrm{M}}$ is the column hold-up volume essential for the determination of the retention factor, $k . R$ is the gas constant and $T$ is the thermodynamic temperature (in Kelvins) [19-21]. By plotting $\ln k$ versus $T^{-1}$ over a sufficiently broad temperature range, it may be calculated the enthalpic and the entropic contributions to retention and selectivity, $-\Delta H^{0}$ from the slope and $\Delta S^{0}$ from the intercept of the plot. van't Hoff plots can provide the information on whether or not the retention mechanisms change over the studied temperature range $[22,23]$. It has been acknowledged that phase transition phenomenon may cause some small deviations from linearity $[24,25]$. That is the consequence of a change in the molecular structure of the stationary phase which appears in the $20-50{ }^{\circ} \mathrm{C}$ range for $\mathrm{C} 18$ silica-based stationary phases. This phenomenon is quite independent of the nature of both solute and eluent. However, it was shown that these deviations were relevant only for stationary phases with high bonding density (bonding densities higher than $4.0 \mu \mathrm{mol} \mathrm{m}^{-2}$ ) [11,25].

Non-linear plots of ln $k$ versus $1 / T$ have been observed more rarely [26, 27]. van't Hoff equation describing curvilinear dependency of $\ln k$ on $1 / T$ can be obtained for the best fit of a curved van't Hoff plot by the partial derivative of $\ln k$ ' with respect to $1 / T$ yielding a second equation (Eq. 3) [28].

$\ln k=b_{0}+\frac{b_{1}}{T}+\frac{b_{2}}{T^{2}}+\ln \Phi$

In this case, both $\Delta H^{0}$ and $\Delta S^{0}$ vary with $T$ according to the following equations:

$\Delta H^{0}=-R\left(b_{1}+\frac{2 b_{2}}{T}\right)$

$\Delta S^{0}=R\left(b_{0}-\frac{b_{2}}{T^{2}}\right)$

$\ln k=-\left(\frac{\Delta G}{\mathrm{RT}}\right)+\ln \left(\frac{V_{\mathrm{S}}}{V_{\mathrm{M}}}\right)$

$b_{0}, b_{1}$ and $b_{2}$ are the parameters of Eq. 3 which can be determined from non-linear regression and consequently used for calculations of system enthalpy, $\Delta H$, and entropy, $\Delta S$. The classical linear van't Hoff behavior is related to a change in the heat capacity of the system independent on $T[29,30]$ and the non-classical van't Hoff behavior to a change in the heat capacity dependent on $T$.

Although RPLC was thoroughly investigated many times, only few works devoted the attention to effect of temperature on retention mechanism especially in low temperatures. These are not commonly used in ordinary highperformance liquid chromatography (HPLC) applications but temperature dependency of retention in broad range can provide description of the energetic principles of chromatographic process.

The goal of this study was to determine the thermodynamic parameter of organic solvents used as organic modifiers in reversed-phase high-performance liquid chromatography (RP HPLC) elution process. The changes of enthalpy 
and entropy on a series of chemically bonded stationary phases were measured to determine, how the temperature and coverage density influence the thermodynamic parameters. The correlation of solvent adsorption values with the enthalpy of solvation was also investigated.

\section{Experimental}

\section{Instruments}

The phases under study were packed into $125 \mathrm{~mm} \times 4.6 \mathrm{~mm}$. stainless steel columns. All columns were packed using a DT 122 packing pump (Haskel, Burbank, CA, USA) under the pressure of $40 \mathrm{MPa}$.

An HP Model 1050 (Agilent Technologies, Waldbronn, Germany) liquid chromatograph was equipped with fourchannel gradient pump, an autosampler with a $100-\mu \mathrm{L}$ loop, diode array UV-Vis detector and computer data acquisition station.

Temperature was controlled using liquid thermostat Julabo F12 (Julabo Labortechnik GmbH, Seelbach, Germany), with the temperature stability $\pm 0.03{ }^{\circ} \mathrm{C}$.

\section{Materials}

The solid support of in-house made phases was Kromasil ${ }^{\circledR}$ 100 (Akzo Nobel, Bohus, Sweden). Five chromatographic columns with different surface coverage densities were studied. All the columns were prepared from the same batch of silica particles. The silica was chemically modified with

Table 1 Coverage density of stationary-bonded phases

\begin{tabular}{llll}
\hline $\begin{array}{l}\text { Phase } \\
\text { code }\end{array}$ & $\begin{array}{l}\text { Carbon } \\
\text { load }\left(P_{\mathrm{c}}\right)(\%)\end{array}$ & $\begin{array}{l}\text { Hydrogen } \\
\text { load }\left(P_{\mathrm{H}}\right)(\%)\end{array}$ & $\begin{array}{l}\text { Coverage density } \\
\left(\alpha_{\mathrm{RP}}\right)\left(\mu \mathrm{mol} \mathrm{m}{ }^{-2}\right)\end{array}$ \\
\hline$\# 1$ & 2.42 & 0.93 & 0.33 \\
$\# 2$ & 7.55 & 1.73 & 1.11 \\
$\# 3$ & 10.88 & 2.24 & 1.68 \\
$\# 4$ & 17.27 & 1.14 & 2.95 \\
$\# 5$ & 18.70 & 3.33 & 3.27 \\
\hline
\end{tabular}

octadecyldimethylchlorosilane (Wacker GmbH, Munich, Germany). Morpholine was purchased from Sigma-Aldrich Chemie (Steinheim, Germany). The characteristics of these stationary phases are listed in Table 1. Columns were not end-capped.

Geometrical parameters of the packed columns and bare silica gel (pore volume, void volume, external porosity and phase ratio) were obtained using inverse size exclusion chromatography [31] and they are listed in Table 2. These data were used for calculation of the phase ratio.

All solvents were HPLC "isocratic grade", purchased from J.T. Baker (Deventer, The Netherlands). Water was purified using Milli-Q system (Millipore, El Paso, TX, USA). All eluents were degassed in ultrasonic bath under vacuum.

\section{Measurements}

Measurements were carried out with flow rate $1 \mathrm{ml} / \mathrm{min}$ at the temperature $295 \mathrm{~K}$. For the temperature dependences, the measurements were carried out in 278, 288, 298, 308, 318 , and $328 \mathrm{~K}\left(5,15,25,35,45\right.$, and $\left.55^{\circ} \mathrm{C}\right)$. The measurements of the solvent retention were repeated in triplicate and arithmetic means of the experimental retention times, $t_{\mathrm{R}}$, and the column hold-up time, $t_{\mathrm{M}}$, were used to calculate the retention factors, $k=\left(t_{\mathrm{R}}-t_{\mathrm{M}}\right) t_{\mathrm{M}}^{-1}$. Organic solvent was injected onto the plateau at given mobile-phase composition. Data were corrected according to the void volume of the apparatus. The highest relative error of retention time measurement was $0.90 \%$, whereas for most measurement it was lower than $0.25 \%$.

The thermodynamic void volume of the column $\left(V_{\mathrm{M}}\right)$ for excess isotherms calculations was obtained by integrating the plot of the retention times of the perturbation peaks $\left(V_{\mathrm{R}}\right)$ from 0 to $100 \%$ of the organic modifier [32]:

$V_{\mathrm{M}}=\frac{1}{C_{\max }} \int_{C=0}^{C=C_{\max }} V_{\mathrm{R}}(C) \mathrm{d} C$

where $C_{\max }$ is a maximum concentration of the organic modifier in the mobile phase.

Table 2 Geometric parameters of the columns measured by inverse size exclusion chromatography

\begin{tabular}{|c|c|c|c|c|c|c|}
\hline $\begin{array}{l}\text { Phase } \\
\text { code }\end{array}$ & $\begin{array}{l}\text { Column void } \\
\text { volume }\left(V_{\mathrm{M}}\right)(\mathrm{mL})\end{array}$ & $\begin{array}{l}\text { Interparticle } \\
\text { volume }\left(V_{\mathrm{ex}}\right)(\mathrm{mL})\end{array}$ & $\begin{array}{l}\text { Pore volume in the } \\
\text { column }\left(V_{\text {pore }}\right)(\mathrm{mL})\end{array}$ & $\begin{array}{l}\text { External } \\
\text { porosity }\left(\varepsilon_{\mathrm{e}}\right)\end{array}$ & $\begin{array}{l}\text { Stationary-phase } \\
\text { volume }\left(V_{\mathrm{s}}\right)(\mathrm{mL})\end{array}$ & $\begin{array}{l}\text { Phase } \\
\text { ratio }\end{array}$ \\
\hline$\# 1$ & 1.711 & 0.878 & 0.833 & 0.42 & 0.365 & 0.213 \\
\hline$\# 2$ & 1.547 & 0.851 & 0.697 & 0.41 & 0.529 & 0.342 \\
\hline$\# 3$ & 1.437 & 0.820 & 0.618 & 0.39 & 0.639 & 0.445 \\
\hline$\# 4$ & 1.251 & 0.778 & 0.473 & 0.37 & 0.826 & 0.659 \\
\hline \#5 & 1.247 & 0.801 & 0.446 & 0.39 & 0.829 & 0.665 \\
\hline
\end{tabular}


Table 3 Parameters $b_{0}, b_{1}$, and $b_{2}$ of Eq. (3) fitted to van't Hoff plot and determination coefficients, $R^{2}$

\begin{tabular}{|c|c|c|c|c|c|c|c|c|}
\hline \multirow{2}{*}{$\begin{array}{l}\alpha_{\mathrm{RP}}^{I} \\
\left(\mu \mathrm{mol} \mathrm{m}{ }^{-2}\right)\end{array}$} & \multicolumn{4}{|c|}{$\mathrm{MeOH}$} & \multicolumn{4}{|l|}{$\mathrm{PrOH}$} \\
\hline & $b_{0}$ & $b_{1}$ & $b_{2}$ & $R^{2}$ & $b_{0}$ & $b_{1}$ & $b_{2}$ & $R^{2}$ \\
\hline 3.27 & -40.8 & $1 \quad 22,813$ & $-3,215,289$ & 0.9929 & -18.52 & 11,409 & $-1,582,457$ & 0.9689 \\
\hline 2.95 & -13.9 & $7 \quad 7,718$ & $-1,086,207$ & 0.9982 & -10.04 & 6,898 & $-982,707$ & 0.9905 \\
\hline 1.68 & -2.1 & $1 \quad 501.9$ & $-34,058$ & 0.9568 & -4.28 & $3,523.6$ & $-518,811$ & 0.8930 \\
\hline 1.11 & -2.1 & 31,095 & $-142,960$ & 0.9735 & -5.63 & 4,097 & $-607,148$ & 0.9512 \\
\hline 0.33 & -6.0 & $6 \quad 2,626$ & $-344,405$ & 0.9962 & -7.13 & 3,963 & $-535,798$ & 0.9926 \\
\hline \multirow{2}{*}{$\begin{array}{l}\alpha_{\mathrm{RP}}^{I} \\
\left(\mu \mathrm{mol} \mathrm{m}{ }^{-2}\right)\end{array}$} & \multicolumn{4}{|l|}{$\mathrm{EtOH}$} & \multicolumn{4}{|l|}{$\mathrm{ACN}$} \\
\hline & $b_{0}$ & $b_{1}$ & $b_{2}$ & $R^{2}$ & $b_{0}$ & $b_{1}$ & $b_{2}$ & $R^{2}$ \\
\hline 3.27 & -30.17 & 17,415 & $-2,452,454$ & 0.9876 & -31.00 & 17,645 & $-2,452,898$ & 0.9949 \\
\hline 2.95 & -12.20 & 7,376 & $-1,044,127$ & 0.9944 & -11.71 & 6,815 & $-921,506$ & 0.9991 \\
\hline 1.68 & 1.86 & $1,039.1$ & $-131,836$ & 0.9899 & -3.85 & $2,014.9$ & $-241,089$ & 0.9962 \\
\hline 1.11 & -2.00 & 1,462 & $-206,583$ & 0.9273 & -2.06 & 1,237 & $-127,311$ & 0.9966 \\
\hline 0.33 & -4.50 & 1,999 & $-230,260$ & 0.9823 & -6.23 & 3,017 & $-369,127$ & 0.9889 \\
\hline
\end{tabular}

In the case of methanol (Fig. 1a), the highest decrease was observed on high coverage density phase $\left(\alpha_{\mathrm{RP}}=3.27 \mu \mathrm{mol} \mathrm{m}{ }^{-2}\right)$ in the range $278-288 \mathrm{~K}$. This decrease was about $30 \%$ of the initial value. For low coverage density phase, the decrease of the adsorbed methanol with increase of the temperature was much smaller. The relative decrease of methanol adsorption in the whole range of tested temperatures $278-323 \mathrm{~K}$ was 55 and $40 \%$ for low coverage phase $(0.33)$ and high coverage phase $\left(3.27 \mu \mathrm{mol} \mathrm{m}^{-2}\right.$ ). It is known that the adsorption of methanol is much weaker than adsorption of acetonitrile [31, 34]. Thus, it can be expected that the temperature influences acetonitrile adsorption much stronger (Fig. 1b). The relative decrease of acetonitrile adsorption in the whole range of tested temperatures $278-323 \mathrm{~K}$ was 70 and $45 \%$ for

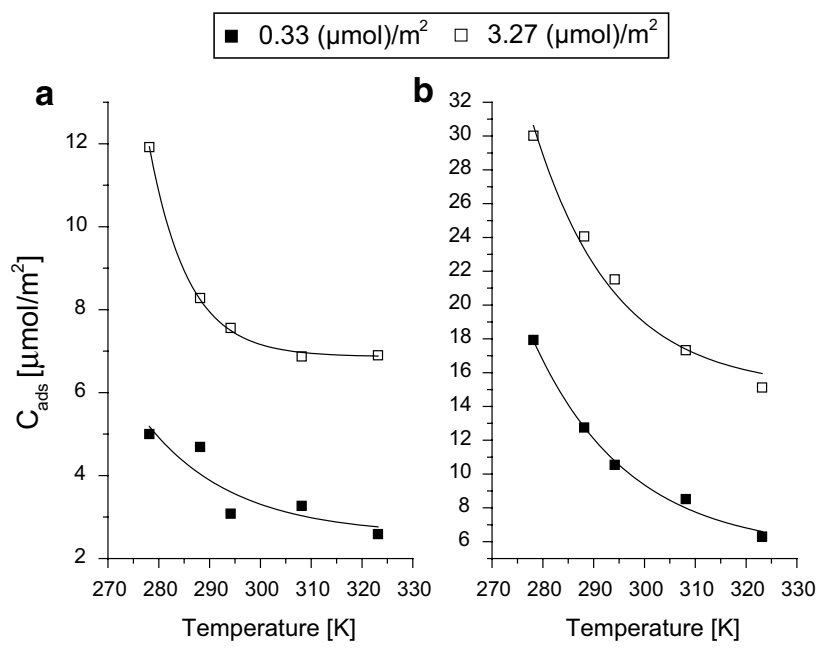

Fig. 1 Influence of the temperature on the maximum concentration of the adsorbed solvent $\left(C_{\text {ads }}\right)$ : methanol (a) and acetonitrile (b). Data according to results presented in [34] 
low coverage phase $\left(0.33 \mu \mathrm{mol} \mathrm{m} \mathrm{m}^{-2}\right)$ and high coverage phase $\left(3.27 \mu \mathrm{mol} \mathrm{m}{ }^{-2}\right)$, respectively. It may be concluded that considerable influence of temperature on adsorption is observed when the modifier strongly solvates the stationary phase. The decrease of solvent adsorption will result in

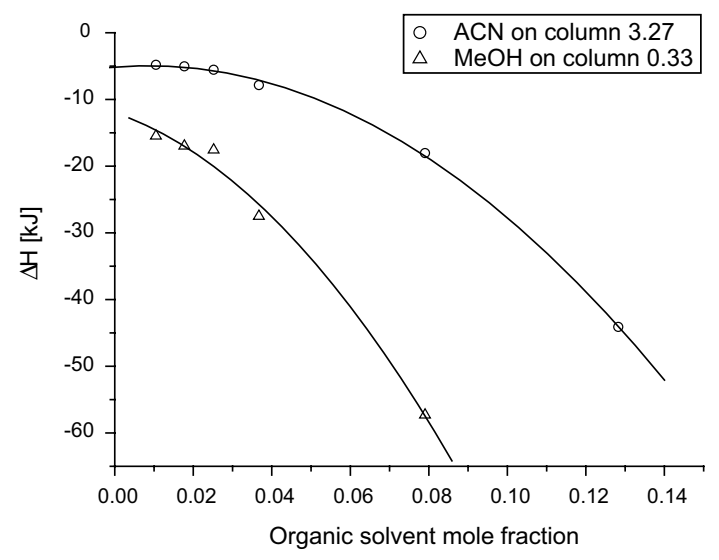

Fig. 2 Changes of the enthalpy of $\mathrm{MeOH}$ and $\mathrm{ACN}$ with mole fraction of organic solvent in the mobile phase at $298.15 \mathrm{~K}$ the decreasing of the elution strength. Thus, the retention of given solute does not changes tremendously with the temperature.

Adsorption of methanol from water solution exhibits higher enthalpy than in the case of acetonitrile, which may be attributed to possibility of hydrogen bond creation, which was proven in the previous study [35]. In Fig. 2, the changes of methanol and acetonitrile enthalpies with the organic solvent mole fraction in the mobile phase at the temperature $298.15 \mathrm{~K}$ are presented. For both solvent, the adsorption process is exothermal. The enthalpy increases (gives more negative values) when the concentration of organic solvent in the mobile phase increases. It is a result of competitive adsorption of water and organic modifiers on the polar residual silanols at stationary-phase surface. When water molecules block most of the polar adsorption sites, the enthalpy of solvent adsorption decreases because organic solvent molecules adsorb mostly on bonded ligands due to weak dispersive forces. Similar results are presented in [36], where water-methanol mixtures provide lower thermal effect (enthalpy) of stationary-phase immersion than pure methanol.

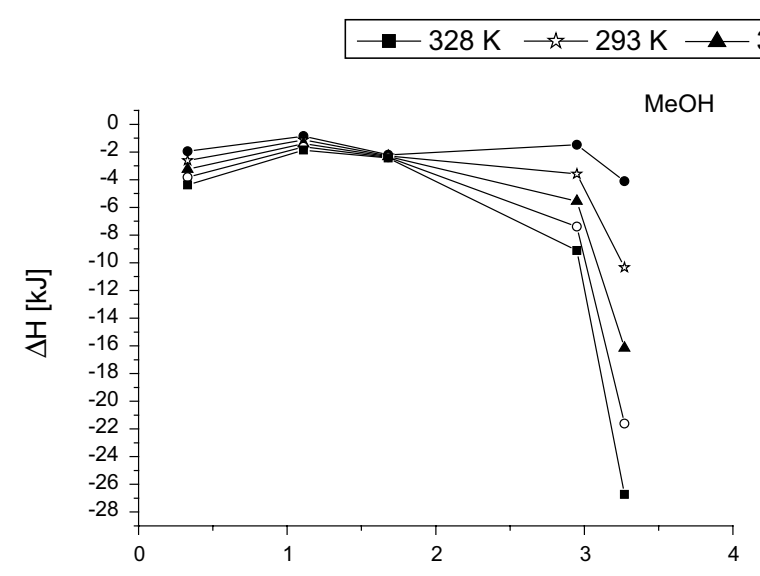

$303 \mathrm{~K} \multimap-0-318 \mathrm{~K} \longrightarrow 283 \mathrm{~K}$
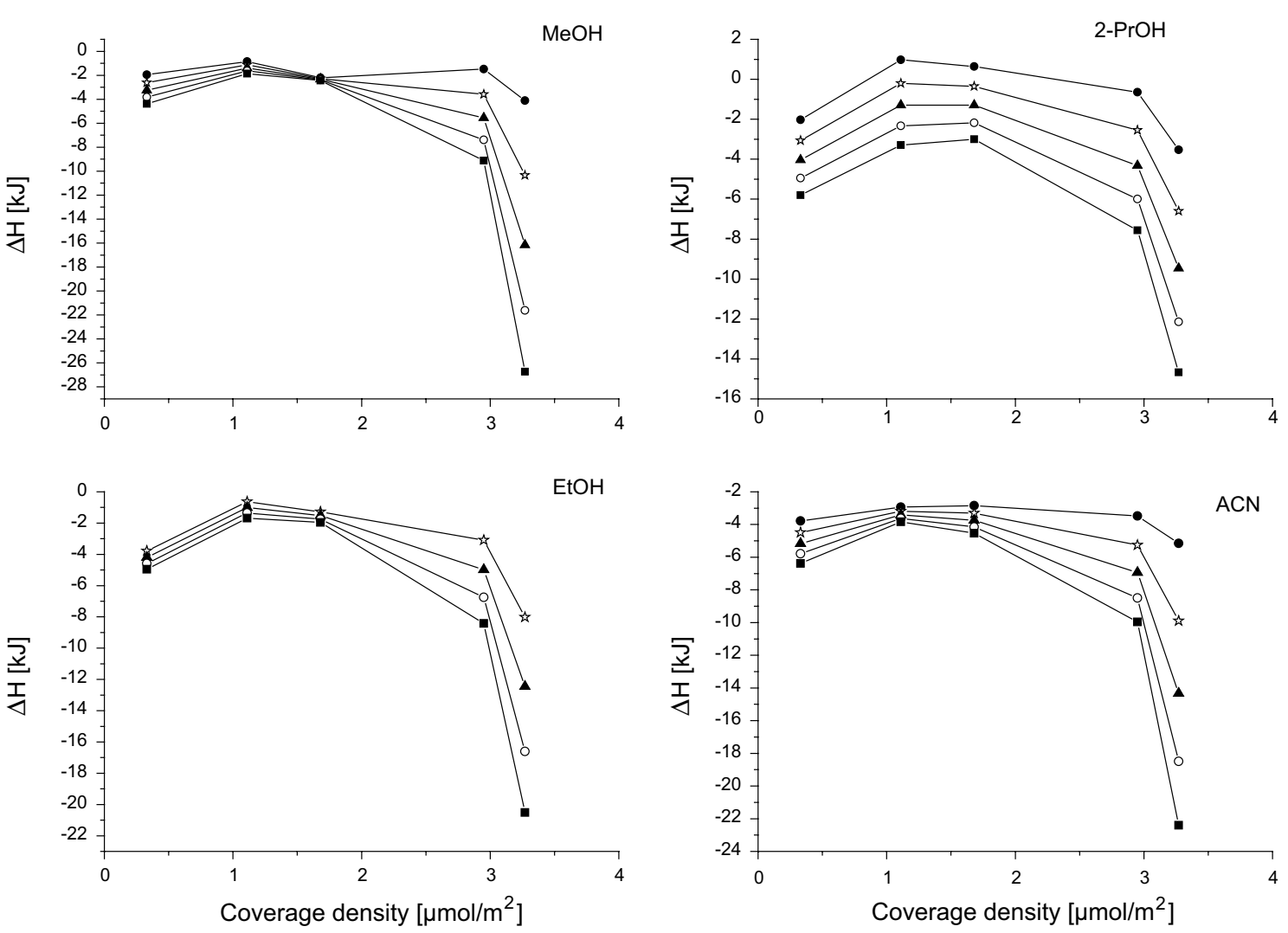

Fig. 3 Changes of the enthalpy of $\mathrm{MeOH}, 2-\mathrm{PrOH}, \mathrm{EtOH}$ and $\mathrm{ACN}$ adsorption with surface coverage density of octadecyl stationary phases measured at $95 \%$ of water in the mobile phase 

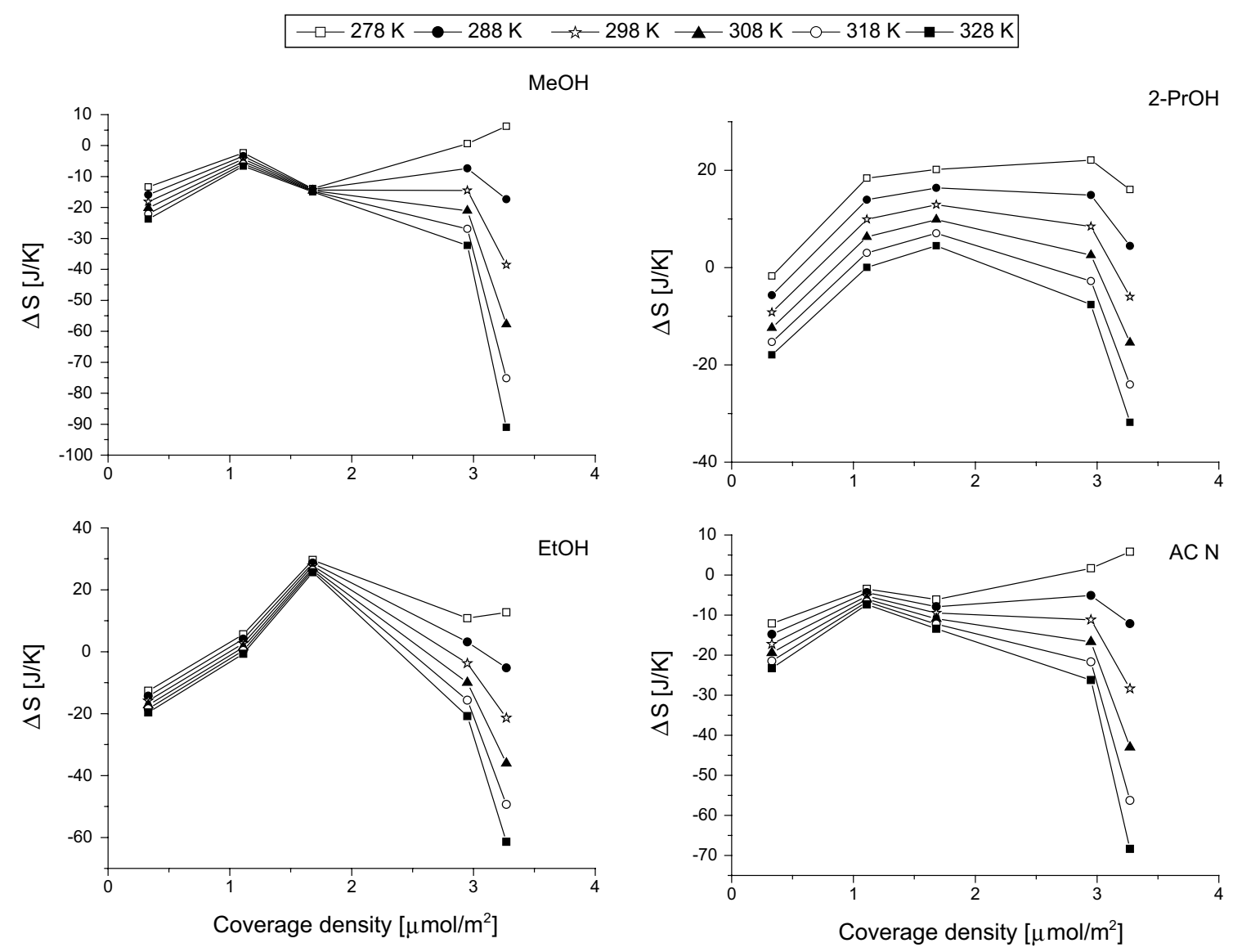

Fig. 4 Changes of the entropy of the $\mathrm{MeOH}, 2-\mathrm{PrOH}, \mathrm{EtOH}$ and $\mathrm{ACN}$ adsorption from water with surface coverage density of octadecyl stationary phases

Changes of the enthalpy and entropy of solvent adsorption were measured using mobile phase containing $95 \%$ of water in the mobile phase. For a series of octadecyl-bonded phases with different coverage densities in the range from 0.33 up to $3.27 \mu \mathrm{mol} \mathrm{m} \mathrm{m}^{-2}$, the enthalpy of all solvents changes in parabolic manner. The lowest values were obtained for stationary phase with $\alpha_{\mathrm{RP}}=1.11 \mu \mathrm{molm}^{-2}$ for all solvents (see Fig. 3). Enthalpies of all solvents on the stationary phase with the lowest surface coverage (and the highest concentration of residual silanols) were slightly higher. Next, the significant increase of the enthalpies was observed, when coverage density of packing materials was extremely high $\left(3.27 \mu \mathrm{mol} \mathrm{m}{ }^{-2}\right)$. The biggest differences are observed between stationary phases 2.95 and $3.27 \mu \mathrm{mol} \mathrm{m}^{-2}$. The reason of such phenomenon may be the change of the interaction mechanism. This stationary phase has extreme coverage density and the migration of solvent molecules between them is hampered. In addition, the competitive adsorption of water is reduced, thus the adsorption of organic solvent is stronger.

Among the four solvents, the highest values of enthalpy $(-\Delta H)$ were observed for methanol-the smallest and the most polar molecule. The lowest enthalpy was measured for propan-2-ol which molecule is the biggest and the most hydrophobic from the tested group. It suggests that solvent enthalpy depends on the polar interaction with residual silanols and on the possibility of penetration between the bonded ligands of the stationary phase that depends on the size of the molecule.

Enthalpies of adsorption from water of all solvents increase with the temperature increase. Also the changes of the solvent enthalpy with surface coverage are more significant at higher temperature. Changes of the enthalpies with temperatures confirm that temperature influences more strongly the solvation of high coverage density phases that is shown in Fig. 1.

Another interesting phenomenon is the similarity of the enthalpies for the stationary phase with coverage density $1.65 \mu \mathrm{mol} \mathrm{m}{ }^{-2}$ at different temperatures, for methanol and ethanol. For this material, the enthalpy is almost independent of the temperature. In the case of acetonitrile, such point is shifted to the material with lower surface coverage $\left(1.11 \mu \mathrm{mol} \mathrm{m}{ }^{-2}\right)$. This situation was not observed for propan-2-ol. 
Parabolic trend of the enthalpy changes may be a result of two different phenomena. First, there are the polar interactions with residual silanols, when the coverage of the bonded ligands is low. When the concentration of bonded ligands increases, the number of residual silanols decreases so the enthalpy decreases as well. However, further increase of the surface coverage density causes the formation of dense bonded layer. Thus, the most dominant process is the solvation of huge number of octadecyl ligands via dispersive interactions. As a result, the enthalpy increases with the increased number of bonded ligands on the stationaryphase surface.

Similar parabolic trend is observed for entropy. The changes of the entropy for all tested solvents in the temperature range from 278 to $328 \mathrm{~K}$ are shown in Fig. 4 . In the case of $\mathrm{MeOH}$ and $\mathrm{ACN}$, the lowest values were obtained for stationary phase with coverage density $1.11 \mu \mathrm{mol} \mathrm{m} \mathrm{m}^{-2}$. However, at $278 \mathrm{~K}$ the minimum is shifted to the highest covered phases. Entropy on low coverage density phases is almost independent of the temperature for $\mathrm{MeOH}$, EtOH and $\mathrm{ACN}$, maximum $10 \mathrm{~J} \mathrm{~K}^{-1}$ in the temperature range from 278 to $328 \mathrm{~K}$. The entropy changes in the case of propan-2-ol are most significant. On the other hand, temperature influences strongly the entropy of $\mathrm{MeOH}, \mathrm{EtOH}$ and $\mathrm{ACN}$ on high covered phases.

It has to be mentioned that entropy for $\mathrm{MeOH}$ and $\mathrm{ACN}$ reaches mostly negative values whereas for $\mathrm{EtOH}$ and 2-PrOH the positive values are observed. In the case of the enthalpy changes, which was shown in Fig. 3, the positive values were observed only for propan-2-ol. From the tested solvent, ethanol and propan-2-ol have the highest hydrophobicity (the longest alkyl chain). A positive $\Delta S$ value indicates a decrease in order of the chromatographic system as the solvent molecule is transferred from the mobile phase to the stationary phase, which is the evidence of the hydrophobic effect [11].

In the case of Gibbs free energy $(\Delta G)$ listed in Table 4, the negative values are observed for $\mathrm{ACN}$, whereas $\mathrm{MeOH}$ provides positive values. The negative values of ACN $\Delta G$ confirm the adsorption process on the stationary phase. The positive values of $\Delta G$ in the case of $\mathrm{MeOH}$ are against adsorption process. However, it has to be remembered that experiments were carried out at high water content in the mobile phase. As it was proven in the previous study [37], adsorption of $\mathrm{MeOH}$ and water is a competitive phenomenon. Adsorption of $\mathrm{MeOH}$ is also much weaker in the comparison with ACN. It has to be mentioned that for the lowest covered stationary phase $\left(0.33 \mu \mathrm{mol} \mathrm{m}{ }^{-2}\right)$, the $\Delta G$ of ACN is also positive that may confirm the theory of significant adsorption of water on the residual silanols and the displacement effect of organic modifier by adsorption of water.

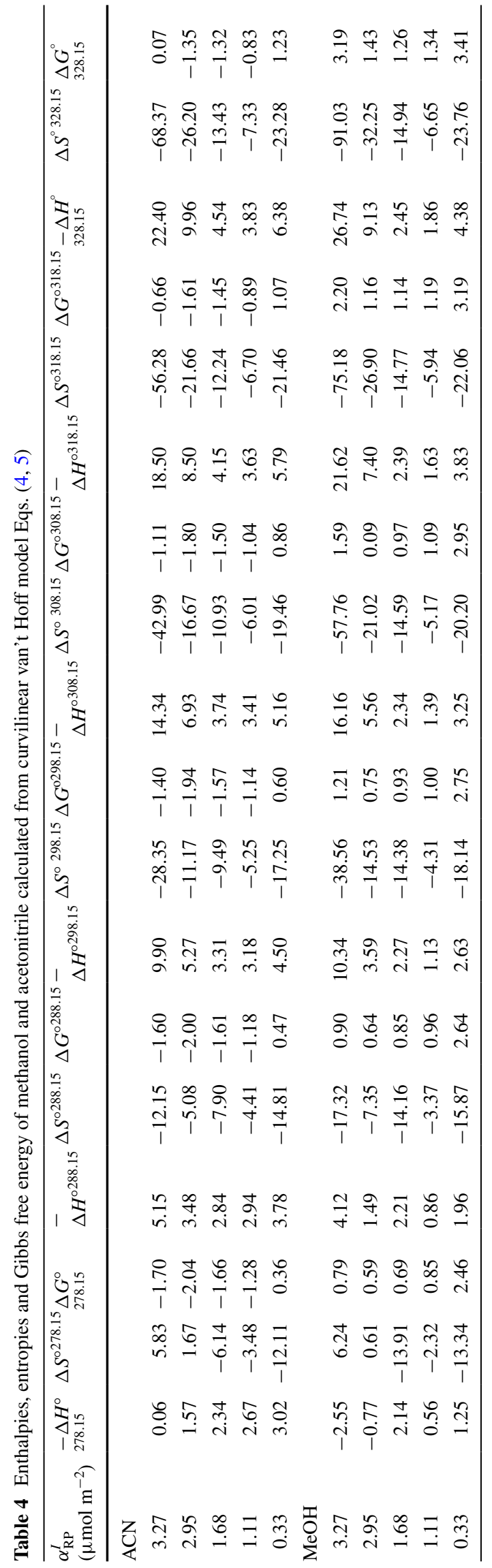



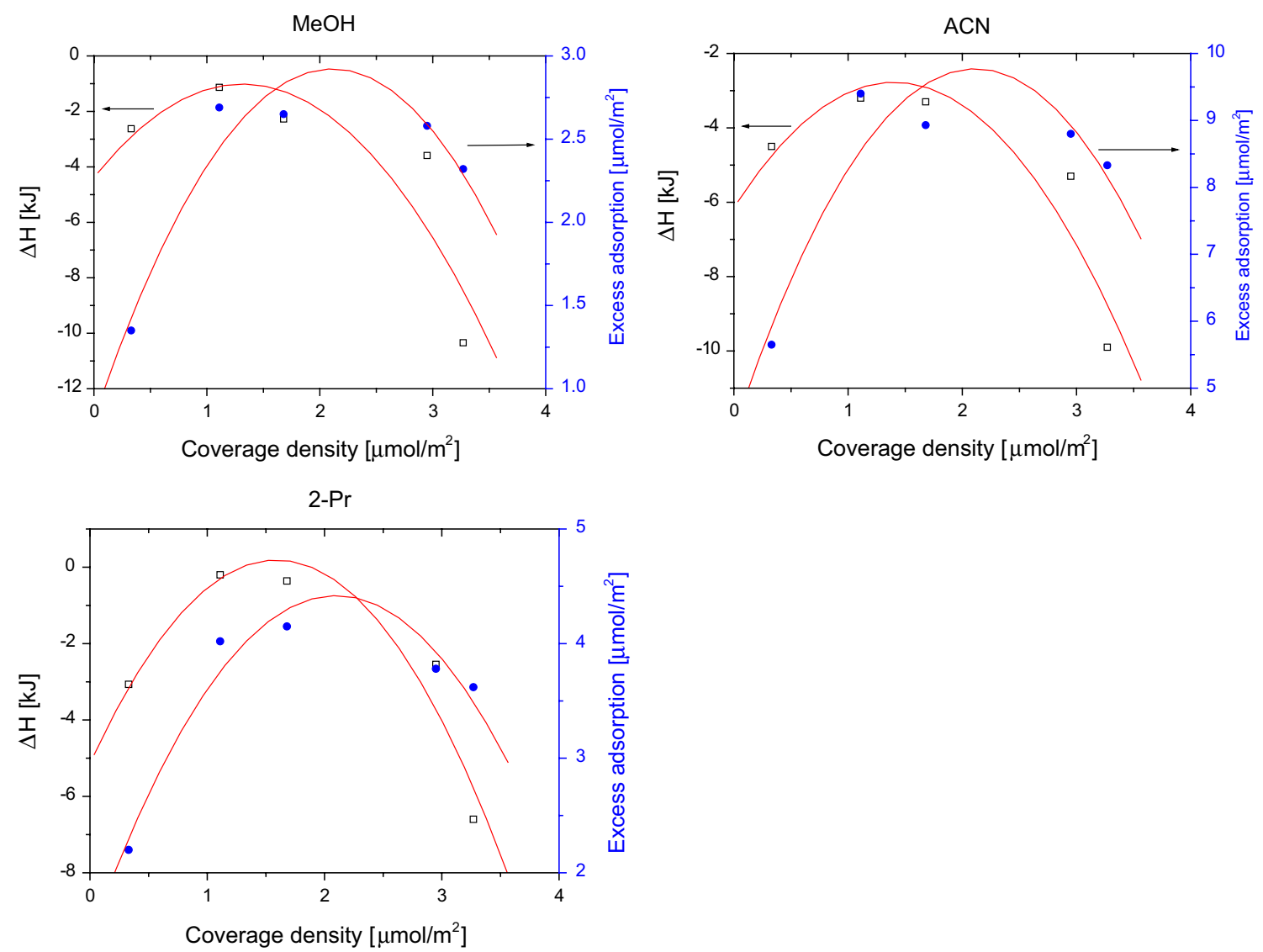

Fig. 5 Changes of the enthalpy and maximum excess adsorbed amount of solvent (maximum of the excess adsorption isotherm measured at $303 \mathrm{~K}$ ) with the coverage density of octadecyl-bonded phases

Comparison of the entropy changes presented in Fig. 4 reveals that the entropy driving solvent molecule into the low coverage density stationary phase is greater than the entropy driving benzene into the high coverage stationary phase. It may indicate a relatively stronger entropic expulsion from the higher density phase, which is consistent with the interphase theory of retention [11] and it was observed in the previous study [38]. The penetration of solvent molecules through a dense film of bonded ligands is hampered in the comparison with low surface coverage phase.

Changes of the enthalpy and entropy over the coverage density of octadecyl phases exhibit similar trend to variation of organic solvent adsorbed amount. As it was discussed in the previous study, as the surface coverage increases, the excess amount of adsorbed solvent increases as well until the effect of the decreasing surface area caused by surface modification becomes significant. Then, due to hampered penetration through the bonded ligands, the amount of adsorbed solvent decreases. Solvent molecules adsorb only on the top of the ligand, however, further increase of surface coverage causes that the portion of solvent molecules is distributed between mobile phase and octadecyl-bonded layer due to partition mechanism [31].
The similarity of enthalpy and adsorption values is compared in Fig. 5. As was discussed above, the higher values of enthalpy are observed, when the adsorption of given solvent increases. For the stationary phases with medium surface coverage, the lowest values of both parameters are observed.

\section{Conclusions}

Temperature is important parameter which influences the retention and solvation in liquid chromatography system. The increase of the temperature causes the decrease of the retention and adsorption of organic solvent used as a organic modifier of the mobile phase. Changes of the enthalpy are related with the variation of amount of adsorbed solvent. The highest values are observed for extreme low and high coverage density phases and the minimum values were measured for stationary phase with medium surface coverage. Entropy of solvation process changes in similar manner to enthalpy.

Both the enthalpy and entropy increase at higher temperature. The changes of entropy and enthalpy with 
temperature increase are the highest for high coverage density phases. Once again, the enthalpy and entropy on stationary phases with medium surface coverage are almost independent on the temperature. Thus, it seems that temperature has weak influence on the solvation and retention on medium covered phase in the comparison with high density phases, where temperature has important influence on both, solute retention and solvation process.

Acknowledgments This work was sponsored by Ministry of Science and Higher Education, Grant No. NCN 2013/09/D/ ST4/03807 for period 2014-2016, the project Enhancement of R\&D Pools of Excellence at the University of Pardubice, reg. Nr. CZ.1.07/2.3.00/30.0021 and CEEPUS scholarship CII-PL-0004-061011-M-43061. The authors thank Akzo Nobel (Bohus, Sweden) for kind donation of the Kromasil 100 silica gel used in this study.

Conflict of interest The authors declare no conflict of interest.

Open Access This article is distributed under the terms of the Creative Commons Attribution License which permits any use, distribution, and reproduction in any medium, provided the original author(s) and the source are credited.

\section{References}

1. Jaroniec M (1993) Partition and displacement models in reversedphase liquid chromatography with mixed eluents. J Chromatogr A $656: 37-50$

2. Slais K, Kreci M (1974) Vacant peaks in liquid chromatography. J Chromatogr 91:161-166

3. McCormick RM, Karger BL (1980) Distribution phenomena of mobile-phase components and determination of dead volume in reversed-phase liquid chromatography. Anal Chem 52:2249-2257

4. Sandi A, Szepesy L (1999) Evaluation and modulation of selectivity in reversed-phase high-performance liquid chromatography. J Chromatogr A 845:113-131

5. Gritti F, Guiochon G (2005) Influence of the pressure on the properties of chromatographic columns III. Retention volume of thiourea, hold-up volume, and compressibility of the C18-bonded layer. J Chromatogr A 1075:117-126

6. Buszewski B, Jaroniec M, Gilpin RK (1994) Influence of eluent composition on retention and selectivity of alkylamide phases under reversed-phase conditions. J Chromatogr A 668:293-299

7. Cole LA, Dorsey JG (1992) Temperature dependence of retention in reversed-phase liquid chromatography. 1 Stationary-phase considerations. Anal Chem 64:1317-1323

8. Horvath C, Melander W, Molnar I (1976) Solvophobic interactions in liquid chromatography with nonpolar stationary phases. $\mathrm{J}$ Chromatogr 125:129-156

9. Melander WR, Horvath C (1980) In: Horvath C (ed) High-performance liquid chromatography, advances and perspectives, vol 2. Academic Press, New York, pp 113-319

10. Dill KA (1987) The mechanism of solute retention in reversedphase liquid chromatography. J Phys Chem 91:1980-1988

11. Cole LA, Dorsey JG, Dill KA (1992) Temperature dependence of retention in reversed-phase liquid chromatography. 2 Mobilephase considerations. Anal Chem 64:1324-1327

12. Gritti F, Kazakevich YV, Guiochon G (2007) Effect of the surface coverage of endcapped C18-silica on the excess adsorption isotherms of commonly used organic solvents from water in reversed phase liquid chromatography. J Chromatogr A 1169:111-124
13. Ferrannini E (1988) The theoretical bases of indirect calorimetry: a review. Metabolism 37:287-301

14. Vanhoenacker G, Sandra P (2005) High temperature liquid chromatography and liquid chromatography-mass spectroscopy analysis of octylphenol ethoxylates on different stationary phases. J Chromatogr A 1082:193-202

15. Li J, Carr PW (1997) Effect of temperature on the thermodynamic properties, kinetic performance, and stability of polybutadiene-coated zirconia. Anal Chem 69:837-843

16. Takeuchi T, Watanabe Y, Ishii D (1981) Role of column temperature in micro high performance liquid chromatography. J High Resolut Chromatogr 4:300-302

17. Greibrokk T, Andersen T (2003) High-temperature liquid chromatography. J Chromatogr A 1000:743-755

18. Škeř́ková V, Jandera $P$ (2010) Effects of the operation parameters on hydrophilic interaction liquid chromatography separation of phenolic acids on zwitterionic monolithic capillary columns. J Chromatogr A 1217:7981-7989

19. Jandera P, Colin H, Guiochon G (1982) Interaction indexes for prediction of retention in reversed-phase liquid chromatography. Anal Chem 435:435-441

20. Chester TL, Coym JW (2003) Effect of phase ratio on van't Hoff analysis in reversed-phase liquid chromatography, and phaseratio-independent estimation of transfer enthalpy. J Chromatogr A 1003:101-111

21. Vigh G, Varga-Puchony Z (1980) Influence of temperature on the retention behaviour of members of homologous series in reversed-phase high-performance liquid chromatography. J Chromatogr A 196:1-9

22. Melander W, Campbell DE, Horvath C (1978) Enthalpy-entropy compensation in reversed-phase chromatography. J Chromatogr 158:215-225

23. Kiridena W, Poole CF, Koziol WW (2003) Reversed-phase chromatography on a polar endcapped octadecylsiloxane-bonded stationary phase with water as the mobile phase. Chromatographia 57:703-707

24. Nahum A, Horvath C (1981) Surface silanols in silica-bonded hydrocarbonaceous stationary phases: I. Dual retention mechanism in reversed-phase chromatography. J Chromatogr 203:53-63

25. Morel D, Serpinet J (1981) Influence of the liquid chromatographic mobile phase on the phase transitions of alkyl-bonded silicas studied by gas chromatography. J Chromatogr 214: 202-208

26. Hearn MTW, Zhao G (1999) Investigations into the thermodynamics of polypeptide interaction with nonpolar ligands. Anal Chem 71:4874-4885

27. Bell CM, Sander LC, Wise SA (1997) Temperature dependence of carotenoids on $\mathrm{C} 18, \mathrm{C} 30$ and $\mathrm{C} 34$ bonded stationary phases. J Chromatogr A 757:29-39

28. Guillarme D, Heinisch S, Rocca JL (2004) Effect of temperature in reversed phase liquid chromatography. J Chromatogr A 1052:39-51

29. Cole LA, Dorsey JG (1990) Reduction of reequilibration time following gradient elution reversed-phase liquid chromatography. Anal Chem 62:16-21

30. Tchapla A, Heron S, Colin H, Guiochon G (1988) Role of temperature in the behavior of a homologous series in reversed phase liquid chromatography. Anal Chem 60:1443-1448

31. Bocian S, Vajda P, Felinger A, Buszewski B (2009) Excess adsorption of commonly used organic solvents from water on nonend-capped $\mathrm{C} 18$-bonded phases in reversed-phase liquid chromatography. Anal Chem 81:6334-6346

32. Kazakevich YV, McNair HM (1995) Study of the excess adsorption of the eluent components on different reversed-phase adsorbents. J Chromatogr Sci 33:321-327 
33. Kazakevich YV, LoBrutto R, Chan F, Patel T (2001) Interpretation of the excess adsorption isotherms of organic eluent components on the surface of reversed-phase adsorbents effect on the analyte retention. J Chromatogr A 913:75-87

34. Buszewski B, Bocian S, Zera R (2010) Influence of temperature and pressure on the preferential adsorption of component of hydroorganic mobile phase in liquid chromatography. Adsorption $16: 437-445$

35. Buszewski B, Bocian S, Rychlicki G, Matyska M, Pesek J (2012) Determination of accessible silanols groups on silica gel surfaces using microcalorimetric measurements. J Chromatogr A 1232:43-46
36. Buszewski B, Krupczyńska K, Rychlicki G, Łobiński R (2006) Effect of coverage density and structure of chemically bonded silica stationary phases on the separation of compounds with various properties. J Sep Sci 29:829-836

37. Bocian S, Vajda P, Felinger A, Buszewski B (2010) Effect of end-capping and surface coverage on the mechanism of solvent adsorption. Chromatographia 71:S5-S11

38. Buszewski B, Bocian S, Rychlicki G, Vajda P, Felinger A (2010) Study of solvent adsorption on chemically bonded stationary phases by microcalorimetry and liquid chromatography. J Colloid Interf Sci 349:620-625 